\title{
Instantonic Methods for Quantum Tunneling in Finite Size
}

\author{
Marco Zoli* \\ School of Science and Technology - CNISM, University of Camerino, 62032 Camerino, Italy.
}

(Received on 5 June, 2009)

\begin{abstract}
The instantonic approach for a $\phi^{4}$ model potential is reexamined in the asymptotic limit. The path integral of the system is derived in semiclassical approximation expanding the action around the classical background. It is shown that the singularity in the path integral, arising from the zero mode in the quantum fluctuation spectrum, can be tackled only assuming a finite (although large) system size. On the other hand the standard instantonic method assumes the (anti)kink as classical background. But the (anti)kink is the solution of the Euler-Lagrange equation for the infinite size system. This formal contradiction can be consistently overcome by the finite size instantonic theory based on the Jacobi elliptic functions formalism. In terms of the latter I derive in detail the classical background which solves the finite size Euler-Lagrange equation and obtain the general path integral in finite size. Both problem and solution offer an instructive example of fruitful interaction between mathematics and physics.
\end{abstract}

Keywords: Path Integral Methods, Finite Size Systems, Instantons

\section{Introduction}

The tunneling through a potential barrier plays a central role in many areas of the physical sciences. It is well known that quantum tunneling is an intrinsically nonlinear phenomenon which cannot be attacked by standard perturbative methods. As an example, take a symmetric double well potential (DWP) often encountered in the form of a two level system in condensed matter physics [1-3]: the stable minima are asymptotically connected by the classical (anti)kink solution, a charge conserving domain wall, whose energy is inversely proportional to the quartic force constant $(\delta>0)$ of the $\phi^{4}$ potential at a given vibrational frequency inside the well. Then, even small quartic nonlinearities induce large classical energies which, in turn, enhance the effects of the quantum fluctuations in the overall probability amplitude for a particle to move from one minimum to the next. This explains why standard perturbative techniques fail to describe tunneling processes while semiclassical methods are known to provide an adequate conceptual framework $[4,5]$.

In general, $\phi^{4}$ models also host potentials having a negative sign of the quadratic term $(\delta<0)[6]$. In this case the mid-point of the potential valley would be a classically and locally stable configuration, a metastable state from which the particle would tend to escape via quantum tunneling. In such state, known in quantum field theory as "false vacuum" [7], the system has a finite lifetime due to the presence of a negative eigenvalue in the quantum fluctuation spectrum which governs the decay into the "true vacuum". The classical solution of the Euler-Lagrange equation for metastable potentials is a time-reversal invariant bounce [8] carrying zero charge whose energy is inversely proportional to the anharmonic parameter $\delta$. Then, also analysis of metastable states and decay rates in tunneling problems are unsuitable for perturbative methods.

Semiclassical methods usually extend the WKB approximation [10] and permit to evaluate the spectrum of quadratic quantum fluctuations around the classical background. While such methods had been first applied to infi-

*Electronic address: marco.zoli@unicam. it nite size systems, extensions to finite size systems have been later on developed [11] to obtain correlation functions and spectra of $\phi^{4}$ and other model potentials [12]. Classical mechanical systems [13-15] perturbed by spatio-temporal noise are known to be sensitive to finite size effects and, in particular, there is growing interest in metallic nanowires and micromagnets with magnetization reversal, in view of their technological potential. Intense research is in progress also on biological systems and specifically DNA to establish the finite size effects on the physical properties [16-18].

While a detailed study of decay rates in finite size metastable potentials has been presented in Ref.[19], this paper reconsiders the infinite size instantonic theory for a bistable $\phi^{4}$ potential: in particular, I will point out the feature which justifies a consistent finite size analysis entirely based on the Jacobi elliptic functions formalism. The latter is not exactly well known among the physicists. A stimulating introduction to the elliptic functions has been published by Good [20] while a systematic treatment can be found in the Refs. [21, 22].

Thus, I feel, this is an interesting situation in which physics and mathematics mutually benefit by each other: the well known instanton problem offers the ground for an application of the Jacobian functions while the latter provides the fundamental tool to extend the instanton theory to cases of practical interest.

The key point of the instanton problem can be synthesized as follows: due to the time translational invariance a Goldstone mode appears in the system. As shown in detail below, such mode is proportional to the (anti)kink velocity and represents the ground state for the quantum fluctuation spectrum. Accordingly, the associated zero mode eigenvalue $\varepsilon_{0}$ breaks the Gaussian approximation and makes the Euclidean path integral divergent. The trouble is overcome by regularizing the fluctuations determinant and evaluating the contribution of the zero mode separately. In doing that however, the size $L$ of the system (the period of the instanton along the time axis) is assumed large but finite so that $\varepsilon_{0}^{-1 / 2} \propto L$ and the path integral is finite. Then, to be consistent, also the classical equation of motion should be solved assuming a finite $L$ and the (anti)kink, peculiar of the infinite size theory, should be recovered as a limit case of the more general, finite size (anti)instanton solution. This problem is discussed in detail 
in Section II. In Section III, I revisit the standard Euclidean path integral $[23,24]$ implemented by the functional determinants method which permits to derive, in the asymptotic limit, the contribution stemming from the quantum fluctuations around the (anti)kink $[25,26]$. A consistent extension of the functional determinants theory to the finite size system allows one to obtain the path integral for the finite size instanton. The general path integral formula are given in Section IV while Section V contains some final remarks.

\section{Classical Equation of Motion}

Take a DWP described by:

$$
V(x)=-\frac{M \omega^{2}}{2} x^{2}+\frac{\delta}{4} x^{4}+\frac{\delta}{4} a^{4}
$$

with minima located at $x= \pm a(V(x= \pm a)=0)$ and positive $\delta$ in units $e V \AA^{-4}$. Note that the relation $a=\sqrt{M \omega^{2} / \delta}$ holds thus posing a constraint on the potential parameters. Eq. (1) is equivalent to:

$$
V(x)=\frac{M \omega^{2}}{4 a^{2}}(x-a)^{2}(x+a)^{2} .
$$

At a fixed $a$, a larger oscillator frequency $\omega$ inside the well leads to an increased nonlinearity. Obviously there cannot be classical motion through the barrier of a DWP and, in the real time formalism, the very solutions are given by the vacua: $x_{c l}= \pm a$. However, applying a Wick rotation which maps the real time $t$ onto the imaginary time $\tau$, the DWP is turned upside down and the classical equation of motion admits a non trivial solution [27]. The latter is the classical path $x_{c l}(\tau)$ which minimizes the action in the imaginary time path integral formalism. Let's find it solving the Euler-Lagrange equation which reads:

$$
M \ddot{x}_{c l}(\tau)=V^{\prime}\left(x_{c l}(\tau)\right)
$$

where $V^{\prime}$ means derivative with respect to $x_{c l}$. Integrating Eq. (3), one gets

$$
\frac{M}{2} \dot{x}_{c l}^{2}(\tau)-V\left(x_{c l}(\tau)\right)=E
$$

with integration constant $E$. Physically, we are looking for a path covering the distance between $-a$ and $+a$ in the period $L$. This means that the solution has to fulfill the boundary conditions:

$$
x_{c l}(\tau= \pm L / 2)= \pm a .
$$

Moreover, from Eq. (4) it is easily seen that the relation

$$
\dot{x}_{c l}(\tau= \pm L / 2)=\sqrt{2 E / M}
$$

holds on the $\tau-$ range boundaries. Hence, only (anti)instantons associated to values $E \geq 0$ can describe the physics of the DWP. This marks an essential difference with respect to the bounce solution of the Euler-Lagrange equation for metastable potentials which, in fact, exists only for $E \leq 0$ [19]. Of course there is a deep physical reason behind such difference in sign: the instantons fulfills antiperiodic boundary conditions and carries finite topological charge whereas the bounce is a time reversal invariant object fulfilling periodic boundary conditions.

Integrating Eq. (4), one gets:

$$
\tau-\tau_{0}= \pm \sqrt{\frac{M}{2}} \int_{x_{c l}\left(\tau_{0}\right)}^{x_{c l}(\tau)} \frac{d x}{\sqrt{E+V(x)}} .
$$

$\tau_{0}$ represents the time at which the middle point of the valley in the reversed potential is crossed. As the system is translationally invariant, $\tau_{0}$ can be placed everywhere along the imaginary time axis, $\tau_{0} \in[-L / 2, L / 2]$. This arbitrariness ultimately represents the physical origin of the zero mode in the stability equation which governs the quantum fluctuations spectrum (see below). Eq. (7) admits a solution which interpolates between the vacua providing evidence for the existence of quantum tunneling. Such solution is in fact a family of paths whose shape depends on the associated energy E.

To be specific, it is clear from Eq. (7) that the zero energy configuration $(E=0)$ is consistent with a physical picture in which the particle starts at $x(\tau=-\infty)=-a$ with zero velocity, falls in the valley and reaches the top of the adjacent hill at $x(\tau=+\infty)=+a$ with zero velocity. Integration of Eq. (7) with $E=0$ leads to the classical path:

$$
x_{c l}(\tau)= \pm a \tanh \left[\omega\left(\tau-\tau_{0}\right) / \sqrt{2}\right]
$$

This is the well known (anti)kink solution which exists if and only if the potential minima are asymptotically connected. In the spirit of the Matsubara formalism [24, 28], the period $L$ can be mapped onto the temperature axis as $L=\hbar / K_{B} T$. Hence, for practical purposes, Eq. (8) holds in the low $T$ / large $L$ range whose upper/lower bound is set by $\tau^{*}$ such that $\left|x_{c l}\left(\tau^{*}\right)\right| / a=1$. As long as the latter condition is fulfilled the (anti)kink solution may be assumed as a reliable approximation for the finite size (anti)instanton. This terminology, born in quantum field theory [29, 30], stems from the fact that the tunneling transition happens in a short time, almost instantaneously. From Eq. (8) one also notes that potentials with larger $\omega$ sustain classical kinks in a broader temperature range.

Take now $E>0$ and define:

$$
\begin{aligned}
& \chi_{c l}(\tau)=\frac{1}{\sqrt{2}} \frac{x_{c l}(\tau)}{a} \\
& \kappa=\frac{1}{2}+\frac{2 E}{\delta a^{4}} .
\end{aligned}
$$

Then Eq. (7) transforms into: 


$$
\tau-\tau_{0}= \pm \frac{1}{\omega} \int_{\chi_{c l}\left(\tau_{0}\right)}^{\chi_{c l}(\tau)} \frac{d \chi}{\sqrt{\chi^{4}-\chi^{2}+\kappa / 2}}
$$

which requires some work to be solved in terms of Jacobian elliptic functions. The integral in the r.h.s. of Eq. (10) yields:

$$
\begin{aligned}
& \int \frac{d \chi}{\sqrt{\chi^{4}-\chi^{2}+\kappa / 2}}=\frac{1^{4}}{2} \sqrt{\frac{2}{\kappa}} F(\theta, s) \\
& \theta=2 \arctan \left(\sqrt[4]{\frac{2}{\kappa}} \chi_{c l}\right) \\
& s^{2}=\frac{1}{2}\left(1+\frac{1}{\sqrt{2 \kappa}}\right) .
\end{aligned}
$$

$F(\theta, s)$ is the elliptic integral of the first kind with amplitude $\theta$ and modulus $s$ :

$$
F(\theta, s)=\int_{0}^{\theta} \frac{d \alpha}{\sqrt{1-s^{2} \sin ^{2} \alpha}},
$$

whose quarter-period is given by the first complete elliptic integral $K(s)=F(\pi / 2, s)$ [21]. In terms of $F(\theta, s)$, one defines the Jacobi elliptic functions sn-amplitude, cnamplitude, dn-amplitude :

$$
\begin{aligned}
& s n[F(\theta, s)]=\sin \theta \\
& c n[F(\theta, s)]=\cos \theta \\
& d n[F(\theta, s)]=\sqrt{1-s^{2} \sin ^{2} \theta} .
\end{aligned}
$$

¿From Eqs. (10), (11) one gets:

$$
\pm \sqrt[4]{\frac{\kappa}{2}} 2 \omega\left(\tau-\tau_{0}\right)=F(\theta, s)
$$

and using the relations:

$$
\begin{aligned}
\tan \theta & =\frac{2 \tan (\theta / 2)}{1-\tan ^{2}(\theta / 2)} \\
\tan \theta & =\frac{s n[F(\theta, s)]}{\operatorname{cn}[F(\theta, s)]}
\end{aligned}
$$

Eq. (14) transforms into:

$$
\begin{aligned}
& \frac{s n(2 \Phi, s)}{c n(2 \Phi, s)}=\frac{2 \cdot{ }^{4} \sqrt{\frac{2}{\kappa}} \chi_{c l}}{1-\sqrt{\frac{2}{\kappa}} \chi_{c l}^{2}} \\
& \varpi= \pm^{4} \sqrt{\frac{\kappa}{2}} \omega\left(\tau-\tau_{0}\right) .
\end{aligned}
$$

The condition $4 \sqrt{\frac{2}{\kappa}} \chi_{c l}(\tau) \neq \pm 1$ imposed by Eq. (16) is equivalent to $x_{c l}(\tau) / a \neq \pm\left(2 s^{2}-1\right)^{-1 / 2}$ which is always fulfilled in the physical range of our interest. The 1.h.s. in the first of Eq. (16) can be rewritten using the double arguments relations:

$$
\begin{aligned}
& \operatorname{sn}(2 \varpi, s)=\frac{2 \operatorname{sn}(\varpi, s) c n(\varpi, s) d n(\varpi, s)}{1-s^{2} s n^{4}(\varpi, s)} \\
& c n(2 \varpi, s)=\frac{c n^{2}(\varpi, s)-s n^{2}(\varpi, s) d n^{2}(\varpi, s)}{1-s^{2} s n^{4}(\varpi, s)}
\end{aligned}
$$

and, through the definition in Eq. (9), from Eqs. (16), (17) I derive the two solutions for the classical equation of motion in the finite size model:

$$
\begin{aligned}
& x_{c l}^{(1)}(\tau)=\sqrt[4]{2 \kappa} a \frac{\operatorname{sn}(\varpi, s) d n(\varpi, s)}{c n(\varpi, s)} \\
& x_{c l}^{(2)}(\tau)=-{ }^{4} \sqrt{2 \kappa} a \frac{c n(\varpi, s)}{\operatorname{sn}(\varpi, s) d n(\varpi, s)} .
\end{aligned}
$$

Set now $E=0$ in Eq. (18). For $\kappa=1 / 2, s^{2}=1$ and the elliptic functions reduce to the limit cases [31]:

$$
\begin{aligned}
& \operatorname{sn}(\varpi, 1)=\tanh \varpi \\
& \operatorname{cn}(\varpi, 1)=\operatorname{sech} \varpi \\
& d n(\varpi, 1)=\operatorname{sech} \sigma .
\end{aligned}
$$

Then, from Eq. (18), I get consistently:

$$
\begin{aligned}
& x_{c l}^{(1)}(\tau) \rightarrow \pm a \tanh \left[\omega\left(\tau-\tau_{0}\right) / \sqrt{2}\right] \\
& x_{c l}^{(2)}(\tau) \rightarrow \mp a \operatorname{coth}\left[\omega\left(\tau-\tau_{0}\right) / \sqrt{2}\right] .
\end{aligned}
$$

The first in Eq. (20) is the (anti)kink of Eq. (8) whose center of motion is set at $x_{c l}^{(1)}\left(\tau_{0}\right)=0$. Instead, the second in Eq. (20) is the solution of Eq. (7) fulfilling the condition $x_{c l}^{(2)}\left(\tau_{0}\right)=\infty$. This proves the correctness of the analytical work.

Computation of Eq. (18) is an instructive task. Numerical investigation shows that both backgrounds predict the same physics. This is checked by establishing the one to one correspondence between $E$ and the temperature $T^{*}$ at which the boundary conditions expressed by Eq. (5) are fulfilled: setting a value for $E$, both classical backgrounds fulfill Eq. (5) at the same $T^{*}$.

Note that both solutions present singularities. The zeros of $x_{c l}^{(1)}(\tau)$, occurring at $\bar{\sigma}= \pm 2 n K(s)$ with integer $n$ would be divergencies for $x_{c l}^{(2)}(\tau)$. Viceversa, the zeros of $x_{c l}^{(2)}(\tau)$ at $\bar{\varpi}= \pm(2 n+1) K(s)$ are singular points for $x_{c l}^{(1)}(\tau)$.

For practical purposes, the choice of the first in Eq. (18) turns out to be more convenient. In fact, taking the center of motion at $x_{c l}\left(\tau_{0}=0\right)=0, x_{c l}^{(1)}(\tau)$ properly describes a family of finite size (anti)instantons which are bounded, continuous and odd in the range $\tau \in[-L / 2, L / 2]$.

The boundary conditions (see Eq. (5)) for $x_{c l}^{(1)}(\tau)$ are always fulfilled before encountering the singular points, thus 
the paths are always continuous functions within their oscillation periods [19]. Mathematically this means that, defining $\varpi^{*}= \pm \sqrt[4]{\frac{\kappa}{2}} \omega L / 2$, one finds numerically $\varpi^{*} / K(s)<1$, hence the time period $L$ is covered within a lattice period shorter than $2 K(s)$.

Around $x_{c l}^{(1)}(\tau)$, one can further study the finite size effects on the quantum fluctuation spectrum by means of the theory of the functional determinants. The details are given in Refs.[32] and [33] while, in the next Section, I review the general features of the functional determinants method and evaluate the quantum fluctuations contribution for the case of the infinite size theory.

\section{Quantum Fluctuations around the (anti)kink}

For a particle with mass $M$ moving in a potential $V(x(\tau))$ the probability amplitude to propagate, in the imaginary time $L$, from the initial position $x_{i}$ (at $\left.-L / 2\right)$ to the final position $x_{f}($ at $L / 2)$ is given by the path integral [23]

$$
\begin{array}{r}
<x_{f} \mid x_{i}>_{L}=\int D x \exp \left[-\frac{A[x]}{\hbar}\right] \\
A[x]=\int_{-L / 2}^{L / 2} d \tau\left(\frac{M}{2} \dot{x}(\tau)^{2}+V(x(\tau))\right),
\end{array}
$$

where $A[x]$ is the Euclidean action that weighs the contribution of the generic path $x(\tau) . D x$ is the measure of the path integration [34]. Accordingly, by expanding the Euclidean action in the neighborhood of the classical path, the propagation amplitude for a particle in the bistable quartic potential reads

$$
\begin{aligned}
& <x_{f} \mid x_{i}>_{L}=\exp \left[-\frac{A\left[x_{c l}\right]}{\hbar}\right] \int D \eta \exp \left[-\frac{A_{f}[\eta]}{\hbar}\right] \\
& A\left[x_{c l}\right]=\int_{-L / 2}^{L / 2} d \tau\left(\frac{M}{2} \dot{x}_{c l}^{2}(\tau)+V\left(x_{c l}(\tau)\right)\right) \\
& A_{f}[\eta]=\int_{-L / 2}^{L / 2} d \tau\left(\frac{M}{2} \dot{\eta}^{2}(\tau)+\frac{1}{2} V^{\prime \prime}\left(x_{c l}(\tau)\right) \eta^{2}(\tau)\right) \\
& V\left(x_{c l}(\tau)\right)=\frac{M \omega^{2}}{4 a^{2}}\left(x_{c l}(\tau)-a\right)^{2}\left(x_{c l}(\tau)+a\right)^{2} \\
& V^{\prime \prime}\left(x_{c l}(\tau)\right)=\frac{M \omega^{2}}{a^{2}}\left(3 x_{c l}^{2}(\tau)-a^{2}\right) .
\end{aligned}
$$

$D \eta$, defined below, is the integration measure over the path fluctuations. If $L \rightarrow \infty$, from Eq. (8) and the second of Eq. (22), one obtains the classical path action:

$$
A\left[x_{c l}\right]=\frac{2 \sqrt{2}}{3} \frac{M^{2} \omega^{3}}{\delta} .
$$

This is also the (anti)kink energy in units of $\hbar$ exhibiting the peculiar dependence on $\delta$ emphasized in the Introduction.

Taking the $\delta \rightarrow 0$ limit (for a given $\omega$ ) corresponds to decouple the two potential wells $(a \rightarrow \infty)$. Accordingly the classical energy required to connect the minima becomes infinitely large. A slightly different version of $V\left(x_{c l}(\tau)\right)$ in Eq. (22) is often found in the literature with $\omega$ replaced by $\omega / \sqrt{2}$. In this case the action would be given by Eq. (23) without the $\sqrt{2}$ factor.

The quadratic fluctuations contribution to the Euclidean action is derived by the third and fifth of Eq. (22). Performing an integration by parts on the kinetic term, the quantum fluctuation action transforms into:

$$
\begin{aligned}
& A_{f}[\eta]=\frac{M}{2} \int_{-L / 2}^{L / 2} d \tau\left(-\frac{d^{2} \eta(\tau)}{d \tau^{2}}+\right. \\
& \left.+2 \omega^{2}\left(1-\frac{3}{2} \cosh ^{-2} \varpi\right) \eta(\tau)\right) \eta(\tau) \\
& \varpi=\omega\left(\tau-\tau_{0}\right) / \sqrt{2} .
\end{aligned}
$$

The infinite size background of Eq. (8) has been inserted in Eq. (24). Then, consistently, Dirichlet boundary conditions $\eta(\tau= \pm L / 2)=0$, with $L \rightarrow \infty$, have to be assumed to derive Eq. (24) from Eq. (22). I'll come back to this point later. Expanding $\eta(\tau)$ in a series of ortonormal components $\eta_{n}(\tau)$ with coefficients $\zeta_{n}$,

$$
\eta(\tau)=\sum_{n=0}^{\infty} \varsigma_{n} \eta_{n}(\tau)
$$

one observes that the quantum fluctuation components obey a Schrödinger equation with Rosen-Morse potential

$$
\begin{aligned}
& \hat{O} \eta_{n}(\tau)=\varepsilon_{n} \eta_{n}(\tau) \\
& \hat{O}=-\frac{d^{2}}{d \tau^{2}}+2 \omega^{2}\left(1-\frac{3}{2} \cosh ^{-2} \varpi\right)
\end{aligned}
$$

which admits two bound state eigenvalues $\left(\varepsilon_{0}, \varepsilon_{1}\right)$ and a continuum spectrum $\left(\varepsilon_{n}, n \geq 2\right)$ [4]. In particular, the ground state $\eta_{0}(\tau)$ is the Goldstone mode, discussed in the Introduction, whose eigenvalue is $\varepsilon_{0}=0$.

Thus, after solving Eq. (26), the quantum action formally reads:

$$
A_{f}[\eta]=\frac{M}{2} \sum_{n=0}^{\infty} \varepsilon_{n} \varsigma_{n}^{2}
$$

Note that, dimensionally: $\left[\varepsilon_{n}\right] \equiv\left[\sec ^{-2}\right],\left[\eta_{n}\right] \equiv\left[\sec ^{-1 / 2}\right]$, $\left[\zeta_{n}\right] \equiv\left[\AA \sec ^{1 / 2}\right]$.

Using the measure [26]:

$$
\int D \eta=\aleph \prod_{n=0}^{\infty} \int_{-\infty}^{\infty} \frac{d \varsigma_{n}}{\sqrt{2 \pi \hbar / M}}
$$

and performing Gaussian integrations over the directions $\varsigma_{n}$ one formally obtains the quantum fluctuations contribution to the propagator in Eq. (22): 


$$
\int D \eta \exp \left[-\frac{A_{f}[\eta]}{\hbar}\right]=\aleph\left(\prod_{n=0}^{\infty} \varepsilon_{n}\right)^{-1 / 2}
$$

The normalization constant accounts for the Jacobian in the transformation to the normal mode expansion in Eq. (25).

There is a singularity in Eq. (29) caused by the $\varepsilon_{0}=0$ eigenvalue which breaks the Gaussian approximation. This reflects the arbitrariness in the choice of $\tau_{0}$ in Eq. (8). The latter represents in fact a one parameter family of solutions localized around their time center and, as such, they lack the time translational symmetry of the Hamiltonian. Any solution, in the set given by Eq. (8), can undergo an infinitesimal time translation which corresponds to an infinitesimal shift of $\tau_{0}$. The resulting shifted kink is however degenerate with the original one and therefore such a fluctuation costs no action. This is the physical origin of the zero mode. Accordingly we have the recipe to handle the divergence in Eq. (29): it can be easily seen that $d x_{c l}(\tau) / d \tau$ satisfies the Euler-Lagrange equation and solves the homogeneous differential problem associated to Eq. (26), $\hat{O} \eta_{n}(\tau)=0$. Moreover it has no nodes being $x_{c l}(\tau)$ monotonic. Then, $d x_{c l}(\tau) / d \tau$ is proportional to the Goldstone mode $\eta_{0}(\tau)$ of the ortonormal expansion in Eq. (25).

These physical facts permit to tackle the divergence. In fact the integration measure in Eq. (28) has a contribution along the $\varsigma_{0}$ direction. Thus, transforming from the $\varsigma_{0}$ to the $\tau_{0}$-axis (with the norm of $d x_{c l}(\tau) / d \tau$ as Jacobian) one gets [26]

$$
\begin{aligned}
& \int_{-\infty}^{\infty} \frac{d \zeta_{0}}{\sqrt{2 \pi \hbar / M}} \exp \left(\frac{-M \varepsilon_{0} \varsigma_{0}^{2}}{2 \hbar}\right)=\sqrt{\frac{M}{2 \pi \hbar}} \bar{N}^{-1} \int_{-L / 2}^{L / 2} d \tau_{0} \\
& \left(\varepsilon_{0}\right)^{-1 / 2} \rightarrow \sqrt{\frac{M}{2 \pi \hbar}} \bar{N}^{-1} L \\
& \bar{N}^{-1}=\sqrt{2 \int_{0}^{L / 2} d \tau\left|\dot{x}_{c l}(\tau)\right|^{2}}
\end{aligned}
$$

The replacement $\left(\varepsilon_{0}\right)^{-1 / 2} \rightarrow \sqrt{A\left[x_{c l}\right] / 2 \pi \hbar} L$ is often encountered in the literature. The latter however holds only in the $L \rightarrow \infty$ limit for which $A\left[x_{c l}\right] \equiv M \bar{N}^{-2}$ whereas, for finite size/temperature systems, the replacement in Eq. (30) is correct in general.

Anyway the heart of the matter, unambigously shown by Eq. (30), is that the divergence in the path integral can be removed only by keeping $L$ finite. It can be certainly large but it has to remain finite. On the other hand the (anti)kink solution in Eq. (8) is intrinsically based on the assumption $L \rightarrow \infty$. Moreover, as emphasized above, its derivative $\left(\propto \eta_{0}(\tau)\right)$ satisfies Dirichlet boundary conditions only at $L \rightarrow \infty$. One may face the contradiction by forcing the fluctuation to vanish in a finite $\tau$ - range: this would compress the particle distribution thus lifting the $\varepsilon_{0}$ eigenvalue upwards and removing the zero [8]. Although the discrepancy (with respect to the asymptotic picture) is of order $O(\exp (-\omega L))$ and therefore it may be controlled at large $L$, I felt that there was a need for a consistent formulation of the theory able to incorporate finite size effects already at the classical stage. This justifies the work presented in the previous Section.

Hereafter I complete the derivation of the path integral based on the infinite size (anti)kink. Thus, Eq. (29) can be rewritten as:

$$
\begin{aligned}
& \int D \eta(\tau) \exp \left[-\frac{A_{f}[\eta]}{\hbar}\right]=\frac{F(\omega L)}{\left(\varepsilon_{0} \varepsilon_{1}\right)^{1 / 2}} \frac{\left(\prod_{n=0}^{\infty} \varepsilon_{n}^{\omega}\right)^{1 / 2}}{\left(\prod_{n=2}^{\infty} \varepsilon_{n}\right)^{1 / 2}} \\
& F(\omega L)=\frac{\aleph}{\left(\prod_{n=0}^{\infty} \varepsilon_{n}^{\omega}\right)^{1 / 2}} .
\end{aligned}
$$

Formally, I have multiplied and divided by the product of the harmonic quantum fluctuation eigenvalues $\varepsilon_{n}^{\omega}$ [25]. $F(\omega L)$ is the well known harmonic fluctuation factor which absorbs $\boldsymbol{x}[25]$. The two bound states have been factored out so that the set of continuum eigenvalues (denominator) is discrete but it contains two states less than the harmonic spectrum (numerator). Then, the evaluation of the quadratic quantum fluctuation contribution is practically reduced to the estimate of the ratio of functional determinants in Eq. (31). This has been done by several methods in the literature [3537] extending fundamental ideas by Gelfand and Yaglom [34] and Forman [38]. The fact that a ratio of determinants appears is not a simple mathematical trick. In fact each determinant, being the product over an infinite number of eigenvalues with magnitude greater than one, would diverge in the $T \rightarrow 0$ limit. Such (exponential) divergences cancel out in the ratio which is meaningful both in value and sign and arises naturally in the path integral method [34].

In the instantonic approach, the essential observation is that both determinants satisfy the Jacobi equations (that is, the homogeneous differential equations associated to the respective stability equations) around their classical solutions, see Ref.[26] for an extensive discussion. The remarkable path integral result which follows is that the ratio is determined only on the base of the classical action (or of the classical path velocity) and it yields a factor $9 \omega^{4}$. Then, including also the bound state eigenvalue $\varepsilon_{1}=3 \omega^{2} / 4$ in Eq. (31) and taking the square root of the whole, the fluctuations contribution gets:

$$
\int D \eta(\tau) \exp \left[-\frac{1}{\hbar} A_{f}[\eta]\right]=2 \sqrt{3} \omega F(\omega L) \sqrt{\frac{M \bar{N}^{-2}}{2 \pi \hbar}} L
$$

This result completes the calculation of the quantum fluctuation for the infinite size case in the semiclassical approximation of Eq. (22).

\section{Infinite and Finite Size Path Integrals}

Assume now that, in the propagation amplitude, the initial and final positions are very close to the potential minima: $x_{i} \sim-a, x_{f} \sim a$ and say $\varphi_{0}(x \pm a)$ the ground state 
harmonic wavefunctions in the potential wells. For $x \simeq \pm a$, $\varphi_{0}(x+a) \varphi_{0}(x-a) \sim \sqrt{\frac{M \omega}{\pi \hbar}}$. The harmonic factor, $F(\omega L)$ can be approximated in the large $L$ limit as, $F(\omega L) \sim$ $\sqrt{\frac{M \omega}{\pi \hbar}} \exp (-\omega L / 2)$. Then, from Eq. (22) and Eq. (35), the path propagator for a particle in a single double well potential reads:

$$
\begin{aligned}
& <x_{f} \mid x_{i}>_{L \rightarrow \infty}=\sqrt{\frac{M \omega}{\pi \hbar}} \exp (-\omega L / 2) \Omega_{\infty} L \\
& \Omega_{\infty}=\exp \left[-\frac{A\left[x_{c l}\right]}{\hbar}\right] 2 \sqrt{3} \omega \sqrt{\frac{M \bar{N}^{-2}}{2 \pi \hbar}}
\end{aligned}
$$

This is the general result of the infinite size instantonic theory. The quantum tunneling removes the two fold ground state degeneracy and induces a splitting in the energy levels. The size of this quantum effect is governed by the classical action hosted in the expression of $\Omega_{\infty}$. The classical path solution determines the shape of the potential in the stability equation (Eq. (26)) thus providing the frame within which the quadratic quantum fluctuations display their strength and set the tunneling rate.

Finally and fundamentally, note that $\Omega_{\infty}$ does not depend on temperature in the infinite size instantonic approach as $A\left[x_{c l}\right]$ is a constant in such limit. This limitation of the theory is removed in the finite size method based on the classical background of Section II.

Around such background one has to evaluate the quantum fluctuations contribution by finding the functional determinants ratio for a finite size system [35] after extracting the zero mode as shown above. Working out the calculations, I get the semiclassical path integral for one (anti)instanton in finite size $L$ :

$$
\begin{aligned}
& <x_{f} \mid x_{i}>_{L}=\sqrt{\frac{M}{2 \pi \hbar L}} \cdot \frac{1}{2 \sinh (\omega L / 2)} \Omega(L) L \\
& \Omega(L)=\exp \left[-\frac{A\left[x_{c l}\right]}{\hbar}\right] \cdot \Omega^{Q F}(L) \\
& \Omega^{Q F}(L)=\sqrt{\frac{M \bar{N}^{-2}}{2 \pi \hbar}} \sqrt{\left|\frac{\operatorname{Det}[\hat{h}]}{\operatorname{Det}^{R}[\hat{O}]}\right|}
\end{aligned}
$$

with:

$$
\begin{aligned}
& \operatorname{Det}[\hat{h}]=-4 \sinh ^{2}(\omega L / 2) \\
& \hat{h} \equiv-\partial_{\tau}^{2}+\omega^{2} \\
& \operatorname{Det}^{R}[\hat{O}]=\prod_{n=1}^{\infty} \varepsilon_{n} .
\end{aligned}
$$

Note that the harmonic determinant has been, in turn, normalized over the free particle determinant $\operatorname{Det}\left[-\partial_{\tau}^{2}\right]=$
$\sqrt{\frac{M}{2 \pi \hbar L}}$ which incorporates the constant $\boldsymbol{\aleph}$. In the $L \rightarrow \infty$ limit, I get

$$
\frac{\operatorname{Det}[\hat{h}]}{\operatorname{Det}^{R}[\hat{O}]} \rightarrow 12 \omega^{2}
$$

thus recovering the value of the infinite size instantonic approach given in Eq. (33). This proves the correctness of the analytical procedure.

$\Omega^{Q F}(L)$ in Eq. (34) accounts for the quantum fluctuation effects in the finite size tunneling problem while $\Omega(L)$ is the overall tunneling frequency which removes the twofold degeneracy of the double well potential in a finite domain. From the path integral in Eq. (34) one can extract the physical properties of the closed quantum system for any size.

\section{Final Remarks}

I have reviewed the instantonic method for the propagation amplitude of a particle moving via tunneling in a bistable quartic potential. This has been done within the standard semiclassical scheme for a $\phi^{4}$ model admitting the (anti)kink solution as classical path which interpolates between the vacua in a infinite time. Since the time is conceived as imaginary by a Wick rotation, an infinite time description maps onto the zero temperature limit according to the Matsubara formalism. The classical action and the stability equation for the quantum fluctuations have also been solved in the asymptotic limit. However, in deriving the particle propagator I have pointed out that, at some steps, the time (the size of the system) is in fact assumed to be large but finite in order to tackle the divergency due to the quantum fluctuation zero eigenvalue related to the translational invariance of the system. Led by this observation, I have described the general method which permits a consistent extension of the instantonic approach such to incorporate finite size effects both in the classical background and in the quantum spectrum. The fundamental step, the replacement of the infinite size (anti)kink by the finite size classical path of Eq. (18), has been explained in detail making use of the formalism of the Jacobi elliptic functions. Along similar patterns one generalizes the bounce solution of the Euler-Lagrange equation for metastable potentials to the finite size case [19]. As a general consequence of the finite size formalism, the quantum fluctuation spectrum obtained in semiclassical approximation becomes dependent on the system size or, in the Matsubara formalism, on the system temperature. From a physical point of view, the elliptic functions formalism permits to evaluate the size/temperature effects both on the tunneling energy of bistable potentials and on the decay rate in systems with metastable potentials. 
[3] M. Zoli, Phys.Rev.B 63, 174301 (2001).

[4] L.D. Landau and E.M. Lifshitz, Quantum Mechanics 3rd Edition, Butterworth-Heinemann, Oxford, (1977).

[5] M.V. Berry and K.E. Mount, Rep. Prog. Phys. 35, 315 (1972).

[6] A.M. Sanchez and J.D. Bejarano, J.Phys.A: Math.Gen. 19, 887 (1986).

[7] S. Coleman, Phys. Rev. D 15, 2929 (1977); C.G.Callan and S. Coleman, Phys. Rev. D 16, 1762 (1977).

[8] J.S. Langer, Ann. Phys. 41, 108 (1967).

[9] R. Dashen, B. Hasslacher and A. Neveu, Phys. Rev. D 10, 4114 (1974); ibid. 11, 3424 (1975); ibid. 12, 2443 (1975).

[10] A. Garg, Am.J.Phys. 68, 430 (2000).

[11] M. Lüscher, Commun. Math. Phys. 104, 177 (1986).

[12] G. Mussardo, V. Riva and G. Sotkov, Nucl. Phys. B 670, 464 (2003).

[13] J. Garcia-Ojalvo and J.M. Sancho, Noise in Spatially Extended Systems, Springer, N.Y./Berlin, (1999).

[14] W.G. Faris and G. Jona-Lasinio, J.Phys.A 15, 3025 (1982).

[15] J. Bürki, C.A. Stafford, D.L. Stein, Phys. Rev. E 77, 061115 (2008).

[16] S. Buyukdagli, M. Joyeux, Phys. Rev. E 76, 021917 (2007).

[17] J. Palmeri, M. Manghi, N. Destainville, Phys. Rev. E 77, 011913 (2008).

[18] M. Zoli, Phys. Rev. E, 79, 041927 (2009).

[19] M. Zoli, Eur. Phys. J. B 62, 221 (2008).

[20] R.H. Good, Eur. J. Phys. 22, 119 (2001).

[21] Z.X. Wang and D.R. Guo, Special Functions, Singapore, World Scientific, (1989).
[22] E.T. Whittaker and G.N. Watson, A Course of Modern Analy sis $4^{\text {th }}$ ed. Cambridge University Press, (1927).

[23] R.P. Feynman, Rev. Mod. Phys. 20, 367 (1948) .

[24] R.P. Feynman, A.R. Hibbs, Quantum Mechanics and Path Integrals, Mc Graw-Hill, New York, (1965).

[25] H. Kleinert, Path Integrals in Quantum Mechanics, Statistics, Polymer Physycs and Financial Markets, World Scientific Publishing, Singapore, (2004).

[26] L.S. Schulman, Techniques and Applications of Path Integration, Wiley\&Sons, New York, (1981).

[27] R. Jackiw, Rev.Mod.Phys. 49, 681 (1977).

[28] G.D. Mahan, Many Particle Physics, Plenum Press, New York, (1981).

[29] G.'t Hooft, Phys. Rev. D 14, 3432 (1976).

[30] T. Schäfer, E.V. Shuryak, Rev. Mod. Phys. 70, 323 (1998).

[31] M. Abramowitz and I.A. Stegun, Handbook of Mathematical Functions, Dover Publications, New York, (1972).

[32] M. Zoli, J. Math. Phys. 48, 012111 (2007).

[33] K. Kirsten, A.J. McKane, Ann. Phys. 308, 502 (2003); ibid. J.Phys A: Math.Gen. 37, 4649 (2004).

[34] I.M. Gelfand, A.M. Yaglom, J.Math.Phys. 1, 48 (1960).

[35] A.J. McKane, M.B. Tarlie, J.Math.Phys. 28, 6931 (1995).

[36] M.A. Jafarizadeh, H. Fakhri, Phys. Lett. A 230, 157 (1997).

[37] H. Kleinert, A. Chervyakow, J. Math. Phys. 40, 6044 (1999).

[38] R. Forman, Invent.Math. 88, 447 (1987) ; ibid. Commun. Math. Phys. 147, 485 (1992). 